\title{
Moyamoya Syndrome in an Infant with Aicardi- Goutières and Williams Syndromes: A Case Report
}

\author{
Jagraj S. Brar ${ }^{1,2,3}$ Rahul Verma2,3(-) Mohammed Al-Omari2,3,4 Victoria M. Siu'2,3,5 \\ Andrea V. Andrade $2,3,4 \quad$ Michael T. Jurkiewicz ${ }^{3,6}$ Saptharishi Lalgudi Ganesan 1,2,30
}

${ }^{1}$ Department of Pediatrics, Division of Pediatric Critical Care Medicine, Children's Hospital, London Health Sciences Centre, London, Ontario, Canada

2 Department of Pediatrics, Children's Hospital, London Health Sciences Centre, London, Ontario, Canada

${ }^{3}$ Schulich School of Medicine and Dentistry, Western University, London, Ontario, Canada

${ }^{4}$ Division of Pediatric Neurology, Department of Pediatrics, Children's Hospital, London Health Sciences Centre, London, Ontario, Canada ${ }^{5}$ Division of Medical Genetics, Department of Pediatrics, Children's Hospital, London Health Sciences Centre, London, Ontario, Canada 6 Department of Medical Imaging, Children's Hospital, London Health Sciences Centre, London, Ontario, Canada
Address for correspondence Saptharishi Lalgudi Ganesan, MBBS, MD, DM, Division of Pediatric Critical Care Medicine, Department of Pediatrics, 800 Commissioners Road East, London Health Sciences Centre, London, Ontario, N6A 5W9, Canada (e-mail: Rishi.Ganesan@lhsc.on.ca).

\begin{abstract}
Keywords

- williams syndrome

- moyamoya syndrome

- stroke

- SAM domain and HD domain-containing protein-1

- aicardi-goutières syndrome

Stroke in infancy is a rare phenomenon but can lead to significant long-term disability. We present the story of a 6-month-old Old Order Amish infant with underlying Williams syndrome, a rare neurodevelopmental disorder caused by a microdeletion, encompassing the elastin gene that produces abnormalities in elastic fibers of the lungs and vessels. This infant presented with lethargy, irritability, and a new-onset generalized tonic-clonic seizure. Brain magnetic resonance imaging (MRI) was consistent with ischemic stroke in the supratentorial regions. MR angiogram demonstrated bilateral narrowing of the internal carotid arteries with "ivy sign," suggestive of Moyamoya. Moyamoya disease/syndrome is a cerebrovascular condition that is associated with progressive stenosis of the intracranial vessels and can cause ischemic stroke in young children. Targeted mutation analysis revealed a homozygous c.1411-2A > G splice site variant in the SAMHD1 gene, consistent with a diagnosis of Aicardi-Goutières syndrome type 5 (AGS5), an autosomal recessive condition with multisystem involvement. In our unique case of infantile stroke with Moyamoya syndrome and dual diagnosis of Williams syndrome and AGS5, both diagnoses likely contributed to the cerebrovascular pathology. This case report highlights the importance of suspecting and testing for multiple genetic abnormalities in children presenting with Moyamoya-related stroke.
\end{abstract}

\section{Introduction}

First described in 1957, Moyamoya vasculopathy is characterized by progressive stenosis of the intracranial internal carotid arteries and their proximal branches. ${ }^{1}$ This restric-

received

April 11, 2021

accepted after revision

September 4, 2021

published online

December 1, 2021 tion in blood flow to downstream areas of the brain results in strokes and gradual development of collateral small vessels. On neuroimaging, the appearance of these abnormal lenticulostriate collateral vessels is commonly described as a "puff of smoke," which is translated to Moyamoya in Japanese. (c) 2021. Thieme. All rights reserved.

Georg Thieme Verlag KG,

Rüdigerstraße 14,

70469 Stuttgart, Germany
DOI https://doi.org/ 10.1055/s-0041-1739131. ISSN 0174-304X. 
Ages of presentation are bimodal, with peaks of incidences occurring in children around 5 years of age and adults at 40 years of age. ${ }^{2}$ Due to the impaired cerebral perfusion distal to the stenotic internal carotid arteries, children with Moyamoya vasculopathy are at an increased risk of ischemic stroke and may present with seizures, hemiparesis, dysarthria, aphasia, and cognitive impairment. ${ }^{1,3}$ Moyamoya syndrome (secondary Moyamoya) usually develops secondary to an underlying disorder such as trisomy 21 , sickle cell disease, Williams syndrome (WS), or neurofibromatosis. Herein, we describe a unique case of a 6-month-old infant with ischemic stroke found to have Moyamoya syndrome in the context of rare dual genetic diagnoses occurring in the same child, contributing to her cerebrovascular pathology.

\section{Case Presentation}

A 4-day old female infant, born at 37 weeks' gestation to Old Order Amish parents, was originally seen by medical genetics with regard to intrauterine growth restriction, anemia and thrombocytopenia, small ventricular septal defect, and mild left pulmonary artery stenosis. Chromosome microarray revealed the $1.4 \mathrm{Mb}$ deletion at 7q11.23, consistent with a diagnosis of WS. At 2 months of age, she developed a 2-day history of fever and cough. On arrival, the child was hypoxic and required continuous positive airway pressure. She developed worsening hypoxia and hypercapnia, which prompted endotracheal intubation. There were unexplained episodes of desaturations and bradycardia with poor tidal volumes. Chest computed tomography (CT) imaging revealed collapse of the left upper bronchus upon expiration without extrinsic compression and a hyperlucent left upper lobe herniating across the mediastinum. These findings were consistent with congenital lobar emphysema (CLE). She underwent lobectomy of the left upper lobe and was successfully extubated and discharged home.

At 6 months of age, this infant returned to the emergency room with 2 days of lethargy and irritability. On examination, she was also found to have hypotonia and a head circumference of $42 \mathrm{~cm}$ (30th percentile). Given concerns for meningitis, lumbar puncture revealed cerebrospinal fluid (CSF) with elevated glucose $4.6 \mathrm{mmol} / \mathrm{L}$, elevated protein $523 \mathrm{mg} / \mathrm{L}$, pleocytosis with nucleated cells of $32 \times 10^{6} / \mathrm{L}$, lymphocytes $62 \%$, and erythrocytes of $8 \times 10^{6} / \mathrm{L}$. During her stay, she exhibited generalized tonic-clonic seizures, and treatment for status epilepticus aborted the seizure activity. Brain CT demonstrated extensive areas of ill-defined hypoattenuation and loss of gray-white differentiation in the bilateral middle and anterior cerebral artery territories, with associated local mass effect, suspicious for early ischemic change (- Supplement Figure S1; available online only). Interestingly, foci of parenchymal calcification were also noted adjacent to the right frontal horn within/adjacent to the right head of caudate and in the deep left frontal white matter. The child was managed in the pediatric critical care unit with appropriate neuroprotective measures, including seizure prophylaxis, maintenance of normoglycemia, and targeted temperature management. Brain magnetic resonance imaging (MRI) identified widespread, bilateral, supra- tentorial cortical/subcortical diffusion restriction and edema, greater on the right, concerning for an acute arterial ischemic stroke (-Fig. 1). Intracranial MR angiogram demonstrated narrowing of the internal carotid arteries bilaterally with the "ivy sign," suggestive of sluggish blood flow in Moyamoya syndrome (-Fig. 2); no definite collateral vessels were present. With concern for ischemic stroke and Moyamoya syndrome, the child was started on unfractionated heparin and continued on other neuroprotective interventions. A follow-up brain MRI on day 5 demonstrated extensive bilateral supratentorial strokes involving the frontoparietal regions but sparing the deep watershed white matter. On day 14 of hospitalization, the child was discharged home with persistent left-sided weakness, diffuse hypertonia, and intermittent abduction of both eyes. Notably, follow-up MR angiogram was performed 3 months later, which revealed progression of the internal carotid artery stenosis and the development of subtle collateral vessels (- Supplementary Figure S2; available online only).

Medical genetics consultation was requested. A search using the terms "stroke" and "Amish" in the Amish, Mennonite, and Hutterite Genetic Disorders Database (http://www. biochemgenetics.ca/plainpeople) yielded the possible
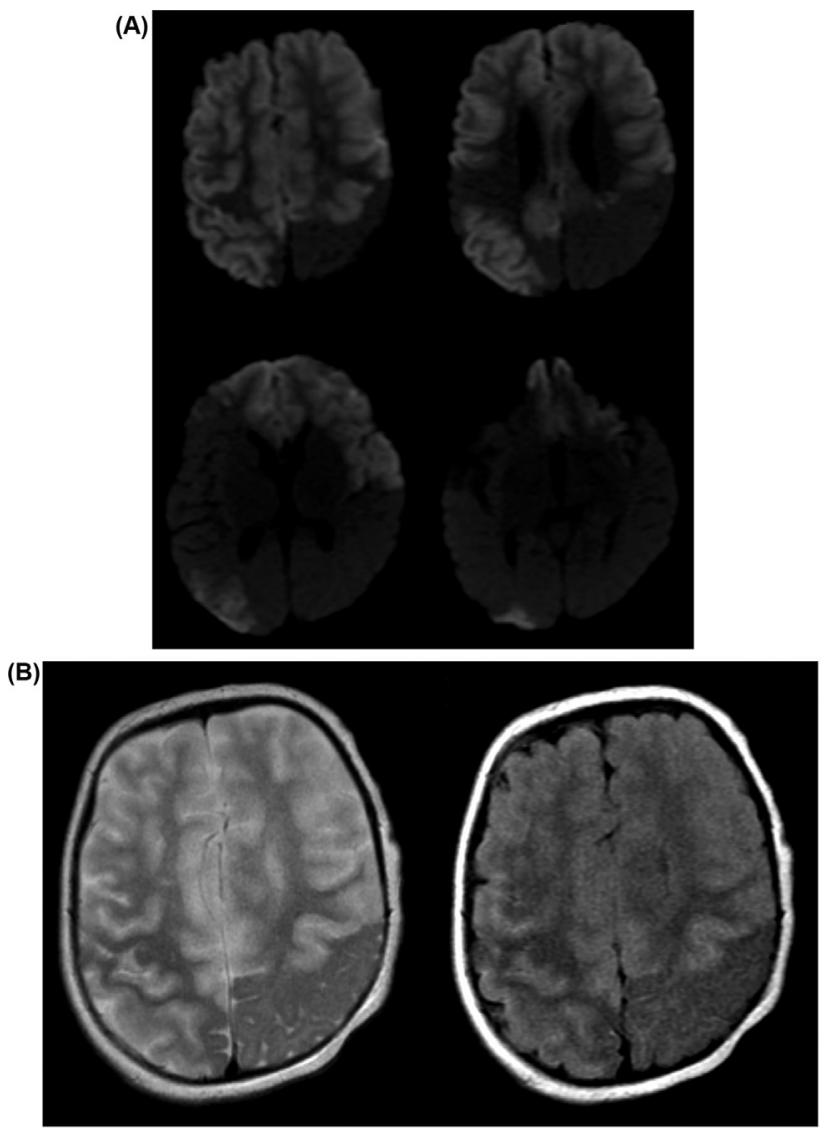

Fig. 1 (A) Axial diffusion-weighted images (DWI). Diffusion restriction involving the entirety of the bilateral frontal lobes and right parietal lobe, and small areas in the right occipital and left parietal lobes. (B) Axial T2-weighted and axial fluid-attenuated inversion recovery (FLAIR) images demonstrate extensive cortical swelling and hyperintensity with abnormal hyperintense signal, extending into the subcortical white matter, corresponding to areas of diffusion restriction (-Fig. 1A), compatible with early ischemic change. 


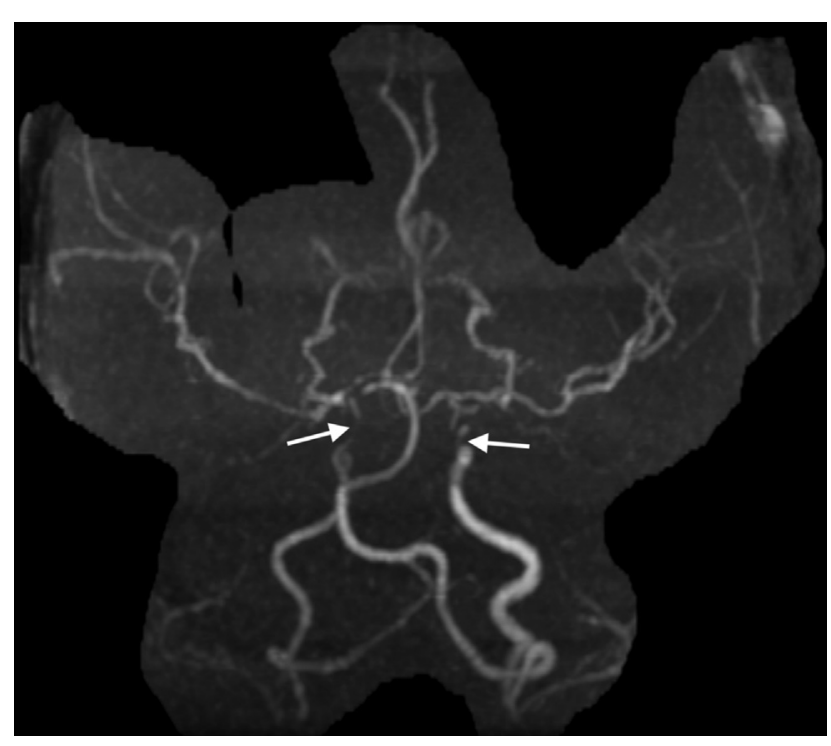

Fig. $23 \mathrm{D}$ reconstructions of time-of-flight magnetic resonance (MR) angiography. Severe narrowing of the cavernous internal carotid arteries is present, more marked on the right, with occlusion in the supraclinoid segment bilaterally (white arrows). No significant collateral vasculature is noted. Posterior communicating arteries are present bilaterally with reconstitution of the internal carotid arteries at this level. The middle cerebral arteries are diminutive bilaterally, smaller on the right.

diagnosis of Aicardi-Goutières syndrome type 5 (AGS5). ${ }^{4}$ Targeted mutation analysis of the SAMHD1 gene (associated with AGS5) for the pathogenic variant known to be present in the Amish community was undertaken. Results confirmed that the child was homozygous for the c.1411-2A $>$ G splice site variant.

\section{Discussion}

We present a unique case of an Old Order Amish infant with dual diagnoses of WS and Aicardi-Goutières syndrome (AGS) with ischemic strokes secondary to Moyamoya syndrome.

First described in 1984, AGS5 is a rare multisystem genetic disorder characterized by a mutation in SAMHD1 gene on chromosome 20q11.23. ${ }^{1}$ AGS5 is associated with intracerebral large artery disease and progressive encephalopathy in infancy, observed with increased frequency in the Old Order Amish population due to founder effect. There are at least seven different genes associated with an AGS presentation, with either autosomal dominant or recessive inheritance pattern. ${ }^{5}$ Our patient presented with nonspecific signs of ischemic stroke consisting of irritability, decreased level of consciousness, and seizure activity. A previous case series of eight patients with AGS found that all had symptoms of acquired microcephaly, spasticity, dystonia, seizures, and rapid deterioration to death. ${ }^{6}$ CT findings of AGS include severe and progressive brain atrophy, intracranial calcifications most commonly localized to the basal ganglia, and deep white matter hypodensities. ${ }^{6-8}$ These image findings were also seen in our patient. CSF findings in our patient were consistent with the typical findings found in AGS of lymphocyte predominance. Persistent CSF lymphocytosis was observed in a review of 27 patients with AGS, suggesting an inflammatory process, with high CSF interferon- $\alpha$ levels. ${ }^{7}$ Cerebral vasculopathy has been described in the literature as a common feature in patients with AGS5 associated with SAMHD1 mutations. ${ }^{9}$ AGS can be associated with pathogenic variants in other genes such as the TREX1 and RNASEH2A/2B; however, these genes have not been associated with large artery disease. ${ }^{9}$

The existing literature is limited with regard to children with AGS5 also experiencing Moyamoya syndrome. Xin et al described a cohort of 14 patients of Old Order Amish with stroke in early infancy and vasculopathy and the same c.1411-2A > G homozygous variant in SAMHD1 as seen in our patient. ${ }^{10}$ Despite significant phenotypic heterogeneity and clinical presentation in their cohort, cerebral vasculopathy was a hallmark with multifocal stenosis in large intracranial arteries, specifically the distal internal carotid artery and proximal middle cerebral artery and Moyamoya pattern. Each patient with stroke in this study initially presented with seizure activity, again similar to our patient who presented in status epilepticus. Dysregulated inflammatory stress response was speculated to be the mechanism of the vascular changes. With regard to treatment options, SAMHD1 has been associated with increased production of proinflammatory cytokines such as interferon- $\alpha 2$, and there may be potential benefit for monoclonal antibodies and other immunosuppressive agents. However, in our patient, we did not initiate treatment specific to SAMHD1 due to the extensive nature of her cerebral infarcts at presentation.

WS is a rare multisystem contiguous gene syndrome typically caused by a 7q11.23 microdeletion, characterized by distinctive facial features, hypercalcemia, and supravalvular aortic stenosis (SVAS). ${ }^{11}$ This deletion includes the elastin (ELN) gene, which is associated with SVAS, peripheral pulmonary stenosis, and cerebral artery stenosis, leading to stroke. ${ }^{11}$ Elastin is a highly cross-linked protein which plays a critical role in tissues which require elastic recoil, such as the lungs, smooth muscle cells in the airways, pulmonary vasculature, and endothelial cells. It has been demonstrated that elastin plays an important role in lung development and conditions, resulting in the loss or destruction of elastin, which is a major factor in lung pathologies including congenital lobar emphysema that this was afflicted with. ${ }^{12}$ Other case reports have also found an association with WS and cerebrovascular stenosis. ${ }^{13}$

Further, a possible association between WS and Moyamoya syndrome has been investigated in only two previous pediatric case reports, in which both patients had a fatal outcome. In the first reported case, an 18-year-old female with WS died of intracerebral hemorrhage due to Moyamoya syndrome. ${ }^{14}$ In the other case, a 9-year-old boy with WS developed a hemiparesis following coarctation repair and was found to have severe stenosis of the bilateral internal carotid arteries and Moyamoya on MRI. ${ }^{15}$ Two days after indirect anastomosis, the child deteriorated with widespread cerebral infarction and subsequently died.

In conclusion, we present a unique case of a young old Order Amish infant with WS and AGS who developed ischemic stroke. A likely explanation is that both the SAMHD1 
mutation associated with AGS and the deletion of the ELN gene seen in WS increased this very young infant's predisposition to cerebral vasculopathy. We emphasize that an atypical age of onset of vasculopathy and stroke should prompt further workup for underlying etiology. Further, patients of Old Order Amish descent with signs and symptoms of stroke should be tested for AGS5. To the best of our knowledge, this is the first case report of a patient with two rare genetic diagnoses that increased the risk of cerebral vasculopathy and stroke.

\section{Author Contributions}

All authors participated in creating content for the manuscript, editing, and provided final approval for submission. No undisclosed authors contributed to the manuscript.

\section{Funding}

The authors have no financial relationships relevant to this article to disclose.

\section{Conflict of Interest}

None declared.

\section{References}

1 Scott RM, Smith ER. Moyamoya disease and moyamoya syndrome. N Engl J Med 2009;360(12):1226-1237

2 Duan L, Bao XY, Yang WZ, et al. Moyamoya disease in China: its clinical features and outcomes. Stroke 2012;43(01):56-60

3 Goswami M, Pandian R, Sharma S. Moyamoya disease-"A puff of smoke": a rare pediatric case report. Int J Clin Pediatr Dent 2020; 13(05):566-568
4 Payne M, Rupar CA, Siu GM, Siu VM. Amish, mennonite, and hutterite genetic disorder database. Paediatr Child Health 2011; 16(03):e23-e24

5 AICARDI-GOUTIERES SYNDROME 5; AGS5: Online Mendelian Inheritance in Man (OMIM). Accessed March 2, 2021 at: https://www.omim.org/entry/612952?search=aicardi\%20goutieres\&highlight=aicardi\%20goutiere

6 Aicardi J, Goutières F. A progressive familial encephalopathy in infancy with calcifications of the basal ganglia and chronic cerebrospinal fluid lymphocytosis. Ann Neurol 1984;15(01):49-54

7 Goutières F, Aicardi J, Barth PG, Lebon P. Aicardi-Goutières syndrome: an update and results of interferon-alpha studies. Ann Neurol 1998;44(06):900-907

8 Uggetti C, La Piana R, Orcesi S, Egitto MG, Crow YJ, Fazzi E. AicardiGoutieres syndrome: neuroradiologic findings and follow-up. Am J Neuroradiol 2009;30(10):1971-1976

9 du Moulin M, Nürnberg P, Crow YJ, Rutsch F. Cerebral vasculopathy is a common feature in Aicardi-Goutieres syndrome associated with SAMHD1 mutations. Proc Natl Acad Sci U S A 2011;108 (26):E232, author reply E233

10 Xin B, Jones S, Puffenberger EG, et al. Homozygous mutation in SAMHD1 gene causes cerebral vasculopathy and early onset stroke. Proc Natl Acad Sci U S A 2011;108(13):5372-5377

11 Pober BR. Williams-Beuren syndrome. N Engl J Med 2010;362 (03):239-252

12 Mecham RP. Elastin in lung development and disease pathogenesis. Matrix Biol 2018;73:6-20

13 Ardinger RH Jr, Goertz KK, Mattioli LF. Cerebrovascular stenoses with cerebral infarction in a child with Williams syndrome. Am J Med Genet 1994;51(03):200-202

14 Kawai M, Nishikawa T, Tanaka M, et al. An autopsied case of Williams syndrome complicated by moyamoya disease. Acta Paediatr Jpn 1993;35(01):63-67

15 Sim Y-W, Lee M-S, Kim Y-G, Kim D-H. Unpredictable postoperative global cerebral infarction in the patient of williams syndrome accompanying moyamoya disease. J Korean Neurosurg Soc 2011; 50(03):256-259 